\title{
Does Intrathecal Baclofen Have a Place in the Treatment of Painful Spasms in Friedreich Ataxia?
}

\author{
Shala Ghaderi Berntsson ${ }^{a} \quad$ Anders Holtz $^{b} \quad$ Atle Melberg $^{a}$ \\ Departments of ${ }^{a}$ Neuroscience, Neurology and ${ }^{b}$ Neuroscience, Neurosurgery, \\ Uppsala University, Uppsala, Sweden
}

\section{Key Words}

Friedreich ataxia $\cdot$ Spasms $\cdot$ Intrathecal baclofen

\begin{abstract}
We present the case of a 50-year-old female patient with Friedreich ataxia (FA) who was treated successfully with an intrathecal baclofen (ITB)-delivering pump for painful spasms. To our knowledge, this is the second reported case of FA where ITB relieved painful and disabling spasms. We suggest that ITB should be considered in the treatment of disabling spasms in patients with FA.

(C) 2013 S. Karger AG, Basel
\end{abstract}

\section{Introduction}

Friedreich ataxia (FA), first described in 1863, is the most common of early-onset hereditary ataxias. This rare autosomal recessive genetic disease is characterized clinically by progressive limb and gait ataxia, dysarthria, areflexia in the lower limbs, sensory loss, muscle weakness and extensor plantar responses [1]. Other major clinical features in FA include cardiomyopathy, scoliosis, swallowing and sphincter disturbance, reduced vision, hearing impairment and diabetes mellitus [2]. However, up to $25 \%$ of the patients with atypical and late-onset phenotypes can present with predominantly spastic ataxia [3]. The discovery of the mutated gene frataxin in 1996 has resulted in a direct molecular diagnostic test looking for GAA repeat expansion in intron 1 of the frataxin gene [4]. Progressive pyramidal degeneration is a common feature in FA, leading to muscular weakness and extensor plantar reflexes. Some patients present with hyperreflexia and spasticity [5]. Intrathecal baclofen therapy (ITB) is an established treatment for severe spasticity of both spinal and cerebral origin. The main group treated with ITB consists of patients with spinal

Shala Ghaderi Berntsson

Department of Neuroscience, Neurology

Uppsala University

SE-75185 Uppsala (Sweden)

E-Mail shala.berntsson@hotmail.com 
cord injury, multiple sclerosis and cerebral palsy; however, less is known about the effect of ITB in other conditions such as FA.

The study was approved by the institutional review board, and written informed consent was obtained from the patient prior to participation.

\section{Case Presentation}

A 50-year-old female was diagnosed with FA in 1993, at the age of 31. At the age of 16, she was referred from the school physician to a pediatrician for the first time because of a subtle gait ataxia. Neurological examination revealed areflexia, muscular hypotonia, reduced position and vibration sense in the lower limbs. A few years later, she presented with an aggravated spastic gait ataxia, postural ataxia and dysarthria as well as urinary and fecal incontinence. At the age of 30 , the patient was no longer able to walk and has been confined to a wheelchair ever since. A few years later, she underwent colectomy and received a suprapubic catheter. She started taking idebenone, a drug that has shown a positive effect on neurological functions in patients with FA [6]. Her parents were first cousins. The diagnosis of FA was confirmed genetically by a DNA analysis showing that the patient was homozygous for an expansion of GAA in the frataxin gene corresponding to 900 repeats. The serum $\alpha$-tocopherol level was normal.

In August 2010, the patient presented to the Department of Neurology, Uppsala University, for her annual visit. She reported frequent and very painful flexor spasms in the lower limbs and trunk. Her sleep was interrupted because of such spasms awakening her throughout the night. Changing the position of her body in the bed or wheelchair, exposing herself to the cold, laughing, coughing, sneezing or taking a shower elicited the spasms, which at times lasted for more than $1 \mathrm{~h}$. Different analgesic drugs including oxycodone, diclofenac and pregabalin were tried without any effect. The combination of oral baclofen in a high dose of $80 \mathrm{mg}$ per day and botulinum toxin injections in the adductor muscles did not improve her painful spasms.

On examination, the patient had a pronounced dysarthria, upper and lower limb ataxia, bilateral foot drop, absent tendon reflexes in all four limbs, and the plantar reflexes were extensor. There was a loss of proprioception and vibration sense in the lower limbs. Muscle tone was not increased in the limbs, and the strength in her arms was preserved. According to the modified Ashworth score, the patient had no spasticity. The spasms were provoked by hip flexion and stretching the legs and lasted for a few minutes, causing great distress to the patient.

The patient received an ITB test injection of $25 \mu \mathrm{g}$ on the first day of testing. The response was remarkable: the painful spasms totally disappeared only $1 \mathrm{~h}$ after the injection, and this positive response lasted for $7 \mathrm{~h}$. During this period, we tried to provoke the spasms by changing her body position, stretching her legs and exposing her to the cold, but none of these maneuvers led to spasms or pain. No side effects were noted during the testing procedure, and we decided to implant an ITB-delivering pump. For the past 2 years, our patient has been on continuous ITB at a constant dose of $100 \mu \mathrm{g}$ per day with preserved efficacy. She experienced great relief of her symptoms and was very satisfied with the result. 
Ghaderi Berntsson et al.: Does Intrathecal Baclofen Have a Place in the Treatment of Painful Spasms in Friedreich Ataxia?

\section{Discussion}

We observed a good and lasting clinical effect on painful spasms in a patient with FA treated with ITB. A similar case of FA with painful spasms in the absence of clinical signs of spasticity was also treated successfully with ITB [7]. The effect of ITB on sleep and respiratory function was investigated by the same authors, and the results showed improved sleep continuity without affecting respiratory function [8]. ITB is traditionally used to reduce severe spasticity, which applies to increased resistance to muscle stretching because of upper-motor neuron lesions [9]. However, disabling spasms due to spinal automatism, as occasionally seen in patients with FA, require more specific therapy at the spinal level. This can be obtained by intrathecal baclofen, a GABA-receptor agonist with an inhibitory effect on peripheral flexor reflex afferents. Being the most frequently inherited ataxia, FA has a prevalence of 1:50,000 [10]. The treatment of patients with FA and other hereditary ataxias is usually symptomatic, and we suggest that ITB should be considered in the clinical setting to relieve the patients' pain and discomfort.

\section{References}

1 Harding AE: Friedreich's ataxia: a clinical and genetic study of 90 families with an analysis of early diagnostic criteria and intrafamilial clustering of clinical features. Brain 1981;104:589-620.

2 Delatycki MB, Corben LA: Clinical features of Friedreich ataxia. J Child Neurol 2012;27:1133-1137.

-3 Bhidayasiri R, Perlman SL, Pulst SM, Geschwind DH: Late-onset Friedreich ataxia: phenotypic analysis, magnetic resonance imaging findings, and review of the literature. Arch Neurol 2005;62:1865-1869.

4 Campuzano V, Montermini L, Molto MD, Pianese L, Cossee M, Cavalcanti F, et al: Friedreich's ataxia: autosomal recessive disease caused by an intronic GAA triplet repeat expansion. Science 1996;271: 1423-1427.

5 Pandolfo M: Friedreich ataxia: the clinical picture. J Neurol 2009;256(suppl 1):3-8.

6 Di Prospero NA, Baker A, Jeffries N, Fischbeck KH: Neurological effects of high-dose idebenone in patients with Friedreich's ataxia: a randomised, placebo-controlled trial. Lancet Neurol 2007;6:878-886.

7 Ben Smail D, Jacq C, Denys P, Bussel B: Intrathecal baclofen in the treatment of painful, disabling spasms in Friedreich's ataxia. Mov Disord 2005;20:758-759.

8 Bensmail D, Quera Salva MA, Roche N, Benyahia S, Bohic M, Denys P, et al: Effect of intrathecal baclofen on sleep and respiratory function in patients with spasticity. Neurology 2006;67:1432-1436.

-9 Lance JW: The control of muscle tone, reflexes, and movement: Robert Wartenberg Lecture. Neurology 1980;30:1303-1313.

10 Ribai P, Pousset F, Tanguy ML, Rivaud-Pechoux S, Le Ber I, Gasparini F, et al: Neurological, cardiological, and oculomotor progression in 104 patients with Friedreich ataxia during long-term follow-up. Arch Neurol 2007;64:558-564. 